Conclusions EBM+ is shown to provide a promising programme for making better use of existing evidence when assessing interventions and exposures.

Concerns about infeasibility, bias and subjectivity can be allayed, although some attempts to tackle these concerns are unsuccessful.

\section{A MULTIFACETED, CLINICALLY INTEGRATED EVIDENCE BASED MEDICINE CURRICULUM IMPROVES MEDICAL STUDENTS' COMPETENCY AS MEASURED BY THE FRESNO TEST}

${ }^{1}$ Bharathy Kumaravel, ${ }^{1}$ Sagana Ratnakumar, ${ }^{1}$ Holly Jenkins, ${ }^{2}$ Jasmine Hearn, ${ }^{1}$ Claire Stocker, ${ }^{1}$ Samantha Chepkin, ${ }^{1}$ Stewart Petersen. 'University of Buckingham Medical School, Buckingham, UK; ${ }^{2}$ Manchester Metropolitan University, Manchester, UK

\subsection{6/bmjebm-2019-EBMLive.26}

Objectives The University of Buckingham Medical School (UBMS) is an independent medical school in the UK. Following feedback from students that they struggled to understand the relevance of Evidence Based Medicine (EBM) to clinical practice, the EBM curriculum was revised. A longitudinal, competency based, multifaceted, clinically integrated curriculum, with assessments has been designed and implemented. The aim of this study was to assess the effectiveness of the new EBM curriculum to improve students' competency using the validated Fresno test and their attitudes.

Method Blended learning approaches have been incorporated with a mix of didactic lectures, facilitated small group discussions and peer teaching of phase I (years one and two) students of the relevance of EBM to clinical practice by phase II (years three and four) students using their personal experiences in clinical rotations. Formative and summative assessments have been designed to capture written demonstration of EBM knowledge and skills as applied to clinical scenarios in short answer format.

All students from the 2017 cohort that experienced the first iteration of the integrated curriculum were invited to participate. The Fresno test of EBM competence was administered as a formative assessment test before and after the EBM teaching through our virtual learning environment. Selfreported students' attitudes and knowledge of EBM, its relevance to clinical practice were assessed and students were invited to participate in a focus group discussion at the end of EBM teaching.

Results Of the 83 students invited, 31 participated at baseline $(37.3 \%)$ and 55 participated at the end of the study (66.3\%). 18 students attempted the Fresno test at baseline as well as at follow-up. The average score for the test was significantly higher after teaching than at baseline, with the average score increasing by 38.7 marks, from 29.3 at baseline to 68.0 after teaching $(p<0.001)$. Analyzing responses to questionnaires from the same 18 students, showed that compared to baseline, after EBM teaching, a higher proportion of students felt confident in critically appraising journal articles and in formulating clinical questions to search for evidence. Five students participated in the focus group discussion and key themes identified were increased students' perceptions of the relevance of EBM to clinical practice, preference for interactive workshops over didactic lectures for literature searching skills and incorporating EBM teaching across the curriculum.

Conclusions It has been feasible to design and implement a multi-faceted, clinically integrated EBM curriculum in undergraduate medical education. Early evaluation of the curriculum using the Fresno test and focus group discussions has shown an improvement in EBM knowledge, skills and students' perceptions of the clinical relevance of EBM. The Fresno test has been a useful formative assessment to assess medical students' competency in EBM- the first three stepsask, acquire and appraise.

\section{CHILDHOOD CANCER HEALTH OUTCOMES IN EGYPT: TEN-YEAR REAL-WORLD EVIDENCE FROM CHILDREN'S CANCER HOSPITAL 57357 - EGYPT (CCHE) AND COMPARISON WITH RESULTS FROM ENGLAND}

\begin{abstract}
${ }^{1,2}$ Ranin Soliman, ${ }^{1}$ Alaa Elhaddad, ${ }^{2}$ Jason Oke, ${ }^{1}$ Wael Eweida, ${ }^{1}$ Iman Sidhom, ${ }^{1}$ Sonia Mahmoud, ${ }^{1}$ Hany Abdelrahman, ${ }^{1}$ Emad Moussa, 'Mohamed Sedki, ${ }^{1}$ Manal Zamzam, 'Wael Zekri, 'Hanafy Hafez, ${ }^{1}$ Amr AbdAllah, 'Mohamed Fawzy, ${ }^{1}$ Mahmoud Hammad, ${ }^{1}$ Hossam Elzomor, ${ }^{1}$ Sahar Ahmed, 'Madeha Awad, ${ }^{1}$ Sayed Abdelhameed, 'Enas Mohsen, 'Lobna Shalaby, ${ }^{1}$ Nourhan Tarek, ${ }^{1}$ Sherif Abouelnaga, ${ }^{3} \mathrm{Heba}$ Fouad, ${ }^{2}$ Carl Heneghan. ${ }^{1}$ Children's Cancer Hospital 57357 Egypt (CCHE), Cairo, Egypt; ${ }^{2}$ University of Oxford, Oxford, UK; ${ }^{3}$ WHO/Regional Office for the Eastern Mediterranean (EMRO), Cairo, Egypt
\end{abstract}

\subsection{6/bmjebm-2019-EBMLive.27}

Objectives To study childhood cancer survival and health outcomes over the last ten years in one Egyptian hospital CCHE (Children's cancer Hospital 57357 Egypt); determine the variations in survival by demographic, cancer type, and disease severity differences and the reasons behind these variations; and compare childhood cancer survival outcomes with results from England.

Method A retrospective observational cohort study was conducted for children (age 0-18 years) with confirmed cancer diagnosis who presented at CCHE for treatment from 2007 until 2017 and were followed up until July 2018. Confirmed diagnosis of childhood malignancy followed the WHO/ICCC-3 criteria. Patients' demographic data were extracted from hospital-based cancer registry, while disease-related and health outcomes data were extracted from hospital disease-specific registry. Health outcomes included 5-year survival rates, agestandardized mortality rates, and trends in disease relapse/progression. Five-year overall survival was calculated for each childhood cancer type using Kaplan Meier analysis. The 5year overall survival rates at CCHE were compared to population-based 5-year survival of children with cancer in England [2001-2015]. Comparable survival was defined as $<10 \%$ difference and $>10 \%$ as inferior survival. Patients' demographics were described for the full-analysis population, and health outcomes evaluation was done for the evaluable population, based on intention-to-treat analysis.

Results A total of 15,997 children with cancer were analyzed; $58 \%$ were males and $42 \%$ females. Most of the patients $(48 \%)$ were in the youngest age group [0-4 years]. Fifty-nine percent of patients had solid tumors and $41 \%$ had hematologic malignancies. The most common cancers were Leukemia, Lymphoma, CNS tumors, and Neuroblastoma. Survival was calculated for 14,553 patients, representing $92.2 \%$ of full study population. 5-year survival rates at CCHE were comparable for some cancer types; 95.6\% for Hodgkin's Lymphoma; $81 \%$ for Non-Hodgkin's Lymphoma; 92.3\% for Retinoblastoma; $82 \%$ for Renal tumors; $66.4 \%$ for CNS tumors; $65.9 \%$ for Ewing Sarcoma; $86.9 \%$ for Germ cell tumors; 61\% for Rhabdomyosarcoma; $77.1 \%$ for other soft tissue tumors; and 91.2\% for CML. Whereas for other cancer types, overall 